\title{
Hilar tuberculosis in a patient with osteosarcoma
}

\author{
B. CANTWELl $\dagger$ \\ M.R.C.P \\ J. J. Fennelly \\ F.R.C.P.I. \\ J. MASTERSON* \\ F.R.C.R. \\ V. LYNCH** \\ F.R.C.S. \\ Departments of $\nmid$ Medical Oncology, * Radiology and ${ }^{* *}$ Surgery, St Vincent's Hospital, Dublin 4
}

\begin{abstract}
Summary
A case of hilar tuberculosis in a patient with osteosarcoma is described illustrating the importance of accurate diagnosis in this unusual association. This occurrence is discussed in the light of the use of potentially immunosuppressive treatments for osteosarcoma.

\section{Introduction}

Pulmonary metastases are the commonest sites of distant metastases in osteosarcoma. Although cytotoxic chemotherapy after primary surgical treatment of limb osteosarcoma is used widely in an attempt to eradicate micro-metastases, hopes of cure once clinically evident metastases are present are limited. However, long survivals have been reported following resection of pulmonary metastases (Huang, Takita and Douglass, 1978). In cases when thoracotomies have been done for the removal of metastatic disease in patients with a variety of primary neoplasms, non-malignant conditions masquerading as pulmonary metastases have been found (Cahan, Shah and Castro, 1978).
\end{abstract}

\section{Case report}

A 16-year-old male presented in March 1979 with a 3-month history of pain in the right knee region. Examination showed swelling around the right adductor tubercle, and radiographs and subsequent bone biopsy indicated osteosarcoma for which a mid-thigh amputation was done. Three weeks after amputation the patient was referred to hospital for assessment for cytotoxic chemotherapy but it was apparent that abnormal enlargement of the left hilar region was present on chest X-ray (Fig. 1). The abnormal left hilar mass was confirmed by tomography and subsequent CT scanning of the chest. He received one course of chemotherapy with adriamycin and methotrexate in combination and subsequently had a thoracotomy with removal of the left hilar node mass and, although the postoperative period was complicated by a left lower lobe collapse and effusion, he made a good recovery. Pathological examination of the excised nodes revealed tuberculous lymphadenitis with caseation granuloma and no evidence of metastatic osteo- sarcoma. He received one year's treatment wh adjuvant cytotoxic chemotherapy in the form $\bar{D} f$ adriamycin and methotrexate given at montlity intervals, and the adriamycin was stopped after $\sigma^{a}$ total safe cumulative dose had been given. He als received anti-tuberculous chemotherapy with ethambutol, isoniazid and rifampicin. $\mathrm{He}$ is now (January 1981) in excess of 21 months after dianosis and primary treatment and is well, with no evidence of recurrence of osteosarcoma and is treatment.

\section{Discussion}

Although tuberculosis should be considered cause of hilar enlargement, it was not possibe to make an accurate pre-thoracotomy diagnos $\$$ this case. Furthermore, there were no clues in the history or physical examination as to the presene of tuberculosis. In the presence of a concomitat osteosarcoma, metastatic disease would have appeared likely, although lung parenchymal and nबt hilar metastases are the usual mode of presentatiőn of metastatic disease in this condition (Mart et al., 1971). This unusual association between hi Rar tuberculosis and osteosarcoma illustrates the i portance of thoracotomy as a treatment optiôn in patients who have potentially removable int thoracic mass lesions in osteosarcoma.

Not only can this procedure in association wh chemotherapy be worthwhile as a treatment for metastatic osteosarcoma (Huang et al., 1978; Beattie, Martini and Rosen, 1975), but it is i portant, as this case illustrates, to exclude no malignant and treatable conditions. Tuberculofsis mediastinal lymphadenopathy has been noted to simulate metastatic malignant disease (Liu, Fields and Shaw, 1978) and, in a study of mediastinal tuberculosis (Bloomberg and Dow, 1980), a high index of suspicion is urged to diagnose atypical cases. The present case gives added impetus to the statement. In particular the possibility of the administration of potentially immunosuppressive cy 8 toxic chemotherapy in the presence of undiagnosed active tuberculosis will be avoided. 


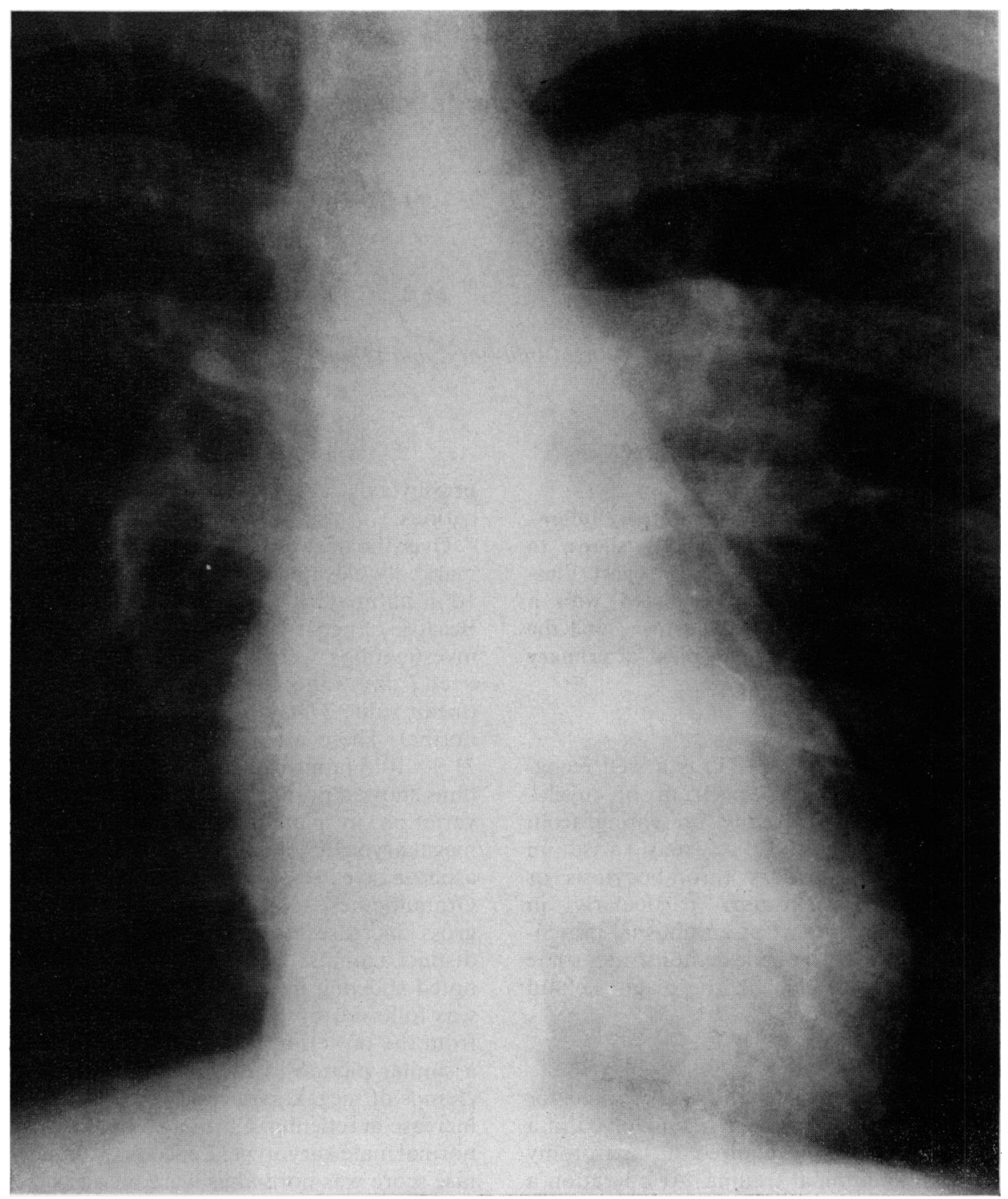

Fig. 1. Smooth lobulated enlargement of the left hilum consistent with hilar glandular enlargement. No other abnormality was shown on conventional whole lung tomography.

\section{Acknowledgment}

We wish to acknowledge the Irish Cancer Society's support for B. Cantwell.

\section{References}

Beattie, E.J., Martini, N. \& Rosen, G. (1975) The management of pulmonary metastases in children with osteogenic sarcoma with surgical resection combined with chemotherapy. Cancer, 35, 618.

BloomberG, T.J. \& Dow, C.J. (1980) Contemporary mediastinal tuberculosis. Thorax, 35, 392.
Cahan, W.G., Shah, J.P. \& Castro, El.B. (1978) Benign solitary lung lesions in patients with cancer. Annals of Surgery, 187, 241.

Huang, M.N., Takita, H. \& Douglass, H.O. (1978) Lung resection for metastatic osteogenic sarcoma. Journal of Surgical Oncology, 10, 179.

LiU, C.-I., Fields, W.R. \& Shaw, C.-I. (1978) Tuberculous mediastinal lymphadenopathy in adults. Radiology, 126, 369.

Martini, N., Huvos, A.G., Mike, V., Marcove, R.C. \& BEATTIE, E.J. (1971) Multiple pulmonary resections in the treatment of osteogenic sarcoma. Annals of Thoracic Surgery, 12, 271. 